\title{
Recent advances in the pharmaceutical manipulation of bone remodelling: the quest for a healthy skeleton
}

\author{
Raubenheimer EJ11, Miniggio $\mathrm{HD}^{2}$, Lemmer $\mathrm{LB}^{3}$, Slavik T4, van Heerden WFP ${ }^{5}$ \\ 1 MChD, PhD, DSc; Ampath Histopathology Laboratory, Pretoria; and Department of Oral Pathology and Oral Biology, University of Pretoria, South \\ Africa \\ 2 BDS, MScMed(Bioethics and Health Law); Unit Oral Biology, Faculty of Health Sciences, Sefako Makgatho Health Sciences University, Pretoria, \\ South Africa \\ MBChB, MMed(Anat Path); Ampath Histopathology Laboratory, Pretoria, South Africa \\ ${ }_{4}$ MBChB, MMed(Anat Path); Ampath Histopathology Laboratory, Pretoria, South Africa \\ ${ }^{5}$ MChD, PhD, DSc; Department of Oral Pathology and Oral Biology, University of Pretoria, Pretoria, South Africa
}

Corresponding author: Prof EJ Raubenheimer, Ampath Histopathology Laboratory, Pretorius Street, Arcadia, Pretoria, South Africa; tel: +27 12 421800; cell: +27 829082788; email: ejraub@fox5.co.za and raubenheimere@ampath.co.za

\begin{abstract}
Introduction: Biochemical characterisation of the autocrine, paracrine and endocrine mediators of bone remodelling provides a scientific basis for the development of pharmaceuticals and autoantibodies which could induce a desired skeletal phenotype. The manipulation of bone remodelling in patients at risk for skeletal disease is gaining clinical momentum due to the benefits of maintaining quality of life rather than restoring the long-term dire consequences of skeletal catabolism.
\end{abstract}

Methods: A narrative review of current literature pertaining to the modes of action of pharmaceuticals and autoantibodies which manipulate skeletal metabolism was performed.

Results: Pharmaceuticals and autoantibodies which manipulate skeletal remodelling can be broadly divided into three categories: bone resorption inhibitors, bone formation stimulators and bone resorption and formation modulators. The mechanisms of action of these medications are briefly summarised and reference is made to the relevant pharmaceuticals and autoantibodies available.

Level of evidence: Level 5

Key words: osteoporosis, skeletal health, osteoinductive medication, antiresorptive medication, bone remodelling

Citation: Raubenheimer EJ, Miniggio HD, Lemmer LB, Slavik T, van Heerden WFP. Recent advances in the pharmaceutical manipulation of bone remodelling: the quest for a healthy skeleton. SA Orthop J 2018;17(1):55-60. http://dx.doi.org/10.17159/2309-8309/2018/v17n1a8

Editor: Prof Anton Schepers, University of the Witwatersrand

Received: June 2017 Accepted: August 2017 Published: March 2018

Copyright: (c) 2018 Raubenheimer EJ, et al. This is an open-access article distributed under the terms of the Creative Commons Attribution Licence, which permits unrestricted use, distribution and reproduction in any medium, provided the original author and source are credited.

Funding: No funding or a gratuity of any kind was received for the study by any of the authors.

Conflict of interest: None of the authors have any interest, directly or indirectly in any of the pharmaceutical companies manufacturing or marketing the products mentioned in the manuscript. 


\section{Introduction}

With an estimated 200 million osteoporotic women in the world and approximately half as many males, the lucrative and yet under exploited market for the prevention of the inevitable end-stage skeletal fractures is evident. ${ }^{1}$ Before the modes of action of bisphosphonates became known, the management of skeletal deficiencies was based on dietary supplementation and control of risk factors for bone catabolism, such as a lack of weight-bearing exercise and the restoration of gonadal hormone deficiencies and mineral homeostasis. Since the 1990s it has progressively become evident that the mediators of the metabolic pathways of bone act on the anabolic and catabolic cell compartments through endocrine, paracrine or autocrine mechanisms, and their outcomes determine the skeletal phenotype. During physiological homeostasis the skeleton is replaced every decade through a process of constant remodelling. This tightly controlled process occurs at defined skeletal sites which are referred to as bone metabolic units or BMUs (the process of bone remodelling is reviewed elsewhere ${ }^{2}$ ). The main goals of bone remodelling are to preserve skeletal strength through constant renewal of bone and maintain vital blood calcium concentrations. Several molecules released during the process impact on systemic metabolism thereby fulfilling the role of the skeleton as an endocrine organ.

Biochemical characterisation of the mediators involved in these complex pathways provides a scientific basis for the development of pharmaceuticals and autoantibodies which can accelerate the formation of bone, prevent bone loss and even revive skeletal health in patients with established skeletal deficiencies like osteoporosis. The long-term effects of manipulating bone remodelling are unknown, as most of the pharmaceuticals and autoantibodies have been in clinical use for less than a decade. As large series of cases accumulate over time, hitherto undescribed complications are certain to be reported with every medication on the market.

For the purpose of this manuscript the pharmaceuticals and autoantibodies which manipulate skeletal remodelling are classified into three main categories: those with antiresorptive properties, those which stimulate bone formation and those with both antiresorptive and bone-forming capacities. This simplified classification is used taking cognisance of the cross-inductive properties of the two cell lines mediating bone remodelling. ${ }^{2}$ Pharmaceuticals and autoantibodies which inhibit bone resorption will invariably also impact on bone formation and vice versa.

It is important to note that before therapy of a compromised skeleton is commenced, all aspects of skeletal metabolism should be optimised including life style and dietary factors. These above-mentioned parameters will not be discussed in this manuscript although they form an important component of the management regimen of the skeletally compromised patient. Furthermore, due to space constraints readers are referred to the recommendations of manufacturers for prescription details of all the pharmaceuticals and autoantibodies mentioned in the manuscript.

The clinical use of these medications will certainly escalate when healthcare providers recognise the benefits of preventing, rather than treating, the dire consequences of a compromised skeleton.

We performed a narrative review of drugs and autoantibodies used in the pharmaceutical management of the diseased skeleton. Readers are referred to the recommendations of the manufacturers for prescription details of the medications mentioned in the manuscript.

\section{Drugs (and autoantibodies) with antiresorptive properties}

\section{Bisphosphonates}

Bisphosphonates (BPs) are biologically analogues of naturally occurring pyrophosphate with which they share an affinity for the surface of hydroxyapatite crystals. Their introduction into the health sciences was initially in dental applications such as the removal of calculus deposits on teeth and caries prevention. ${ }^{3}$ Etidronate, the first commercial BP, was used in the 1960s to treat myositis ossificans. ${ }^{4} \mathrm{~A}$ dose-related response to the inhibition of bone resorption by BPs was demonstrated in Paget's disease of bone in the early 1970 s. ${ }^{5}$ Soon after this discovery the drug became the treatment of choice for skeletal diseases states related to an imbalance of osteoclast-mediated bone resorption such as osteoporosis and malignancy-induced bone disease. The first BP approved for clinical use by the FDA (Food and Drug Administration of the USA) was alendronate in 1995. Today BPs are also used as delivery molecules for $(99 \mathrm{~m})$ Tc-based radiopharmaceuticals during bone imaging. ${ }^{6}$

BPs bind reversibly to the calcium of the hydroxyapatite crystal lattice of bone from where they are released into the environment of the osteoclasts during bone resorption. Their long therapeutic half-life is linked to their slow release and effective suppression of osteoclast activity. The first-generation BPs, also referred to as the simple BPs (etidronate, clodronate, and tiludronate) do not contain a nitrogen ion in their chemical structure and their mode of action is based on the inactivation of ATP (the source of energy for the osteoclast), thereby neutralising osteoclast activity and induction of their premature apoptosis. The more recently developed variants which contain nitrogen ions (zoledronic acid, risedronate, ibandronate, alendronate, neridronate and pamidronate) are more potent osteoclast inhibitors (Table 1). Their mode of action is through a disruption of the synthetic pathways of the cytoskeletal proteins of osteoclasts, thereby impairing their ability to form ruffled borders necessary for their adherence to bone surfaces, which is a requirement for the release of the ions necessary for bone resorption. ${ }^{2,7,8}$ The osteoclasts inhibited by BPs are large and detached from bone surfaces which distinguish them from the attached and smaller osteoclasts seen in hyperparathyroidism.

In addition to their actions on osteoclasts, BPs preserve the viability of bone-forming cells among other mechanisms through their anti-apoptotic effects on osteoblasts and osteocytes. ${ }^{9,10}$ Due to their structure, which is in many aspects similar to the pyrophosphates, several BPs are important inhibitors of mineralisation. Unlike the pyrophosphates, BPs are not neutralised by the alkaline phosphatase produced by osteoblasts. Their potency differs in this regard; however, etidronate inhibits bone resorption and mineralisation at the same concentration and, due to its osteomalacic effect, the drug is not advised for the treatment of osteoporosis. Most of the other nitrogen-containing BPs, however, do not affect mineralisation at therapeutic doses and are therefore recommended as first-line medications for the treatment of osteoporosis. Risendronate and alendronate are the most popular BPs; the latter not only has a high affinity for bone but is also active for longer. ${ }^{1}$ For the prevention of fractures at all skeletal sites, zoledronic acid is the most effective, and zoledronic acid and alendronate were shown to be the most successful combination of BPs for the prevention of hip fracture only. ${ }^{11}$ The choice of a BP however depends on patient tolerance, cost and the medical history of the patient.

The most dramatic application of the BPs is in the management of patients with skeletal metastases. The incidence of pathological fractures, hypercalcaemia and skeletal pain of malignancy are significantly reduced through the offsetting of the bone-resorbing effects of parathyroid hormone-related protein (PTHrP) produced by malignant cells ${ }^{12}$ and the analgesic effects of BPs. 


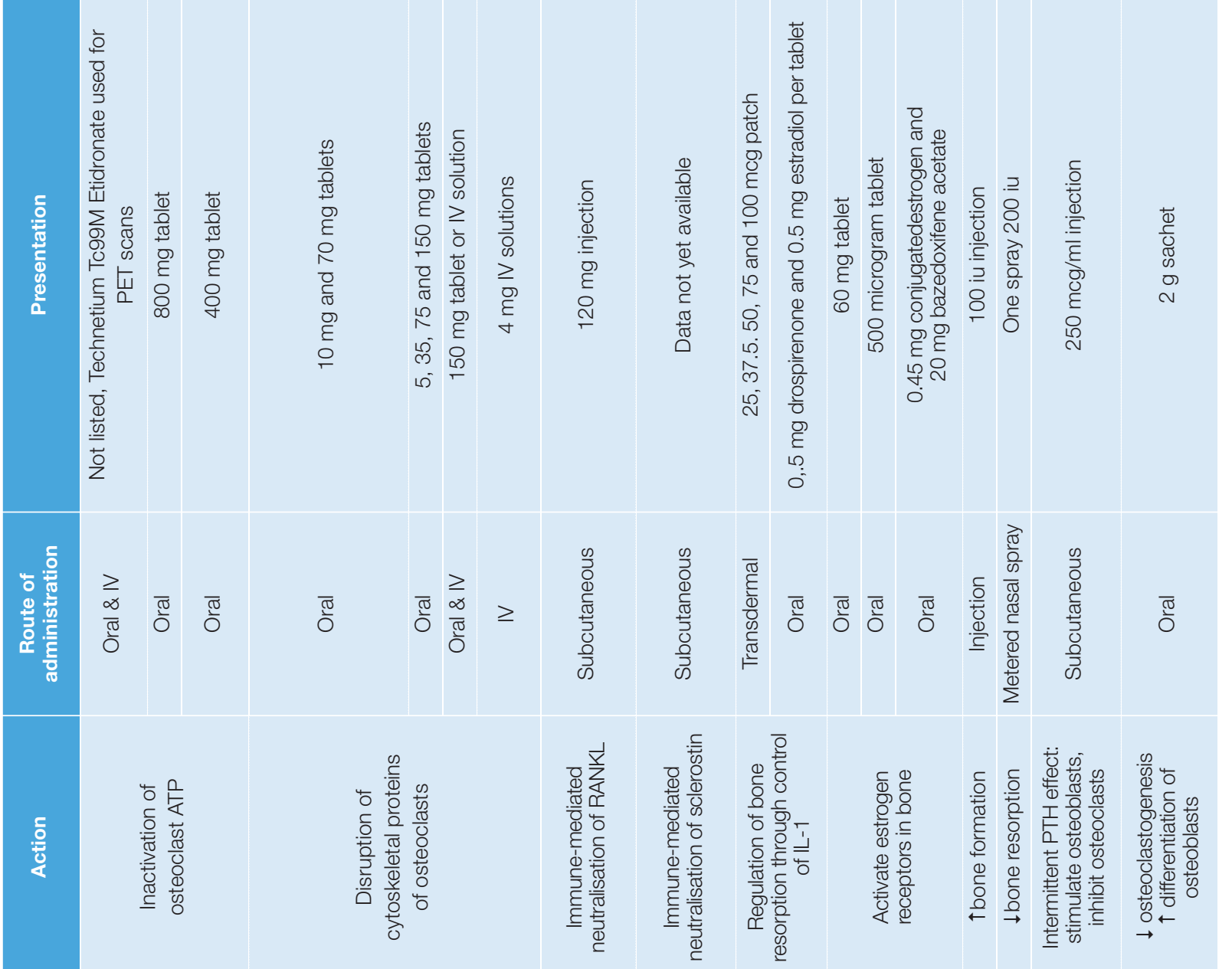


There is substantial evidence in the literature that the fracturerisk reduction of $\mathrm{BPs}$ is highest in women with osteoporosis with probably less benefits accruing in the treatment of other groups, including men. ${ }^{13}$ Current additional approved indications are Paget's disease of bone and osteoporosis associated with glucocorticoid therapy in men. ${ }^{14}$ Treatment indications for Paget's disease are limited to symptomatic disease or biochemically active disease. The drug of choice for the normalisation of bone remodelling is one of the new generation BPs such as risedronate. The drug does not, however eradicate the radiological findings, improve the deformities or reduce pain which results from the associated osteoarthritis. ${ }^{15}$

Atrial fibrillations and other minor adverse effects, such as gastro-oesophageal irritation, have been reported with BP therapy. A small number of patients, particularly those on the first-generation BPs administered intravenously, developed osteochemonecrosis of the jaw bones after invasive dental procedures. ${ }^{16}$ Although the risk of osteochemonecrosis is minimal with orally administered bisphosphonates, surgery exposing bone should be performed under the highest aseptic circumstances possible. Suitable antibiotic cover should be provided only for those patients who receive the drug intravenously. ${ }^{17}$ Atypical subtronchanteric femur fracture is a newly recorded adverse complication of long-term BP therapy. ${ }^{13}$ These fractures are particularly problematic due to their propensity for non-union. With the accumulation of data on long-term use of BPs, more skeletal and extra-skeletal complications are certain to be reported.

Most of the BPs can be administered orally and some (like zoledronic acid) intravenously at intervals of up to one year (Table 1). Oral administration is contraindicated in patients suffering malabsorption syndromes and oesophageal disorders such as achalasia. Generally, patient compliance is better with medications administered parenterally and at longer intervals.

\section{Denosumalb}

The FDA-approved monoclonal antibody denosumab neutralises the receptor activator of nuclear factor- $\mathrm{K} \beta$ ligand (RANKL $)^{2,18}$ and consequently inhibits the activation of osteoclasts, decreases bone resorption and improves bone density. Denosumab is classified as biological (and not pharmacological, thereby bypassing the stringent FDA clearance) and is indicated for the treatment of post-menopausal women with a high risk for osteoporotic fractures or patients who have failed on or are intolerant to other osteoporotic regimens. In a three year study, a relative decrease of $68 \%$ of vertebral and $20 \%$ of non-vertebral fractures were reported in elderly females who received denosumab subcutaneously every six months for 36 months. ${ }^{18}$ These results are similar to those reported for intravenously administered zoledronic acid, and better than those reported for other osteoporotic drugs administered orally. ${ }^{18}$ Denosumab therapy has a risk comparable to the nitrogen-containing BPs for osteochemonecrosis of the jaw bones. ${ }^{16}$ Due to the expression of RANK (receptor activator of NF-K) receptors on immune cells (including dendritic cells), an increased risk of infection, particularly involving the skin, is evidently possible with denosumab therapy. ${ }^{19}$ Adverse reactions such as musculoskeletal pain, hypercholesterolaemia and cystitis have also been reported. ${ }^{20}$ Denosumab is particularly indicated in patients who are intolerant to other anti-osteoporotic drugs, those with renal disease and where patient compliance on drugs requiring daily administration is a problem. As with most of the other pharmaceuticals and autoantibodies, hypocalcaemia and hipovitaminosis D must be corrected before commencement of therapy, and adequate calcium and vitamin D should be administered during treatment.

\section{Hormone replacement therapy (HRT)}

Increased monocyte interleukin-1 (IL-1) in post-menopausal women stimulates bone resorption and contributes significantly to skeletal catabolism. The anabolic effect of HRT on bone occurs mainly through regulation of the release of $\mathrm{IL}-1 .^{2,21} \mathrm{HRT}$ is therefore one of the most successful and costeffective bone saving treatments for women within 10 years after menopause or before 60 years of age. ${ }^{22}$ HRT with standard dose oestrogen in this phase of life has additional benefits of decreasing the occurrence of coronary heart disease; however, it is important that in HRT therapy with oestrogen plus progesterone this benefit is lost.22 Other advantages of oestrogen therapy are improvements of climacteric symptoms and prevention of polyarticular osteoarthritis thereby contributing to a dramatic improvement in quality of life. ${ }^{23}$ Complications of HRT include an increased incidence of venous thrombosis, depression, headaches, breast tenderness, induction of a premenstrual syndrome, skin irritation and weight gain. ${ }^{24} \mathrm{HRT}$ in normal concentrations is however contraindicated for long-term therapy or after the age of 60 years as the increased risk of breast and endometrial carcinoma outweighs the skeletal benefits. ${ }^{25}$ Oestrogen used as a single systemic agent is appropriate in post-hysterectomy women, and the addition of progesterone is advised in patients with an intact uterus due to the danger of endometrial cancer with unopposed oestrogen therapy. After cessation of HRT some females rapidly lose bone mass and follow-up with replacement medication is strongly advised. ${ }^{26}$ Current data does not support the use of HRT in survivors of breast cancer ${ }^{22}$ and therefore other options for the salvage of the skeleton in this patient cohort should be explored.

The use of low dose- and ultra-low-dose HRT has gained popularity over the past years and may provide women aged 60 and older preserved skeletal integrity without the endometrial and breast complications. ${ }^{27}$ Patients on any form of HRT must be regularly examined for breast and endometrial complications. Long-term clinical studies will shed more light on the efficacy and long-term complications of perimenopausal regular dose and post-menopausal low-dose HRT.

\section{Selective oestrogen receptor modulators (SERMs)}

SERMs are compounds that bind to oestrogen receptors and elicit agonist or antagonist responses depending on the prevailing oestrogen milieu. Several SERMs antagonise ER receptors in bone in pre-menopausal women (when oestrogen concentrations are high) but have the opposite effect in the presence of low circulating concentrations of oestrogens. The first SERM developed was tamoxifen which, due to oestrogen-receptor antagonising properties, is the endocrine treatment of choice for advanced breast cancer, adjuvant therapy to surgery, radiation and chemotherapy in the earlier stages of the disease. All SERMs improve axial bone quality and lasofoxifen in particular has been shown to also benefit the quality of trabecular bone in the appendicular skeleton. Both raloxifene and lasofoxifene increase cortical bone thickness and strength. ${ }^{28}$ Complications reported with long-term SERM treatment include increased vaginal discharge, slightly elevated incidence of endometrial cancer (with some SERMs), venous thromboembolism and hot flushes. Indications for the use of SERMs therefore are post-menopausal females (particularly post-hysterectomy patients) and males with osteoporosis. Alternate medication is advised in patients with a tendency towards venous thromboembolism. ${ }^{29,30}$ 


\section{Cathepsin K inhibitors}

Cathepsin $\mathrm{K}$ inhibitors prevent the breakdown of the organic matrix of bone by degrading the proteolytic enzyme cathepsin $\mathrm{K}^{2}$ without interfering with osteoclast activity, as is seen with most other anti-resorptives. ${ }^{31}$ Although preliminary trials indicate that the inhibitors are indeed efficacious, attempts to commercialise the approach were recently aborted. ${ }^{32}$ Following a 12-year clinical trial involving 16000 patients, the pharmaceutical company developing the cathepsin $\mathrm{K}$ inhibitor cited an increased risk of cardiovascular events and suspended further exploitation of this osteoporosis drug. ${ }^{33}$ Extensive long-term clinical trials on this promising approach to the reduction of skeletal catabolism may yield positive results in the future.

\section{Pharmaceuticals with mainly bone forming properties}

\section{Teriparatide}

Intermittent administration of teriparatide, a parathyroid hormone (PTH) derivative, is an FDA-approved anabolic therapy for the restoration of bone health in skeletal deficiency states. ${ }^{34}$ The potent negative regulator of bone formation, sclerostin, is suppressed by intermittent administration of $\mathrm{PTH}$, resulting in a suspension of osteoblast inhibition and subsequent stimulation of bone formation. ${ }^{2}$ Constant elevation of PTH however (as in hyperparathyroidism) has the opposite effect where osteoclast activation is mediated through a facilitation of RANKL and suppression of osteoprotegerin (OPG) production by osteocytes. ${ }^{2,35}$ Several studies in Asia demonstrated that teriparatide is well tolerated and improves lumbar spine bone density while lowering of the incidence of vertebral fractures. Combined treatment with teriparatide and BP does not provide an added beneficial influence on bone mineral densities although a combination with denosumab may have added benefits compared to an individual treatment regimen. ${ }^{36}$ In rats, teriparatide causes an increase in the incidence of osteosarcoma in doses ranging from 3 to 60 times the exposure in humans. Patients with Paget's disease of bone, paediatric populations with open epiphyses and those receiving radiation therapy should not receive teriparatide due to the increased baseline risk of osteosarcoma. Adverse reactions recorded marginally above placebo controls in clinical trials include joint pains, nausea, dizziness, leg cramps and injection-site reactions. ${ }^{37}$

\section{Pharmaceuticals and autoantibodies with antiresorptive and bone forming properties}

\section{Strontium ranelate (SR)}

Strontium is an anion with properties close to those of calcium and is therefore readily incorporated in mineral tissue in place of calcium. Studies have shown that SR modulates the balance between bone resorption and bone formation through stimulation of osteoblast differentiation and survival, ${ }^{38-40}$ reduction of osteoclastogenesis and acceleration of osteoclast apoptosis ${ }^{41}$ through mechanisms which include, among others, the elaboration of prostaglandin $\mathrm{E}_{2}\left(\mathrm{PGE}_{2}\right) .{ }^{42}$ The net result is an increase in bone formation with a simultaneous decrease in bone resorption which correlates with an improvement in the micro architecture of both cortical and trabecular bone. ${ }^{43}$

Until recently, SR was licensed for clinical use in Europe but not in the USA. In 2014 the Pharmacovigilance Risk
Assessment Committee of the European Medicines Agency recommended that restrictions be placed on the use of SR to treat osteoporosis due to its alleged risk for myocardial infarction. SR is cost effective, and according to some studies, safe for the long-term prevention of appendicular and axial fractures in postmenopausal women ${ }^{43,44}$ and men. ${ }^{45}$ The efficiency of the drug is comparable with that of teriparatide and there is no benefit of combining it with another anti-osteoporotic drug. ${ }^{43}$ As a single therapeutic regimen there is good evidence of its tolerability over eight years. It is recommended that SR be taken between meals due to the potential of neutralising absorption from the gastrointestinal tract attributed to binding to ions in the diet. In anticipation of long-term data, the use of SR should be restricted to patients without a risk for cardiovascular events, venous thromboembolism, renal impairment or allergic reactions against the drug. ${ }^{37}$

\section{Serotonin}

The remarkable action of serotonin on the skeleton is related to its bivalent properties. When produced in the brain, it acts as a neurotransmitter and exerts an anabolic effect on the skeleton by enhancing bone formation and inhibiting bone resorption. In contrast, peripherally produced serotonin inhibits bone formation. ${ }^{2,46}$ Ongoing research into drugs that exploit this complicated metabolic axis is likely to contribute to the development of bone-manipulating drugs in the future.

\section{Calcitonin}

Calcitonin will not be covered in detail in this manuscript as it has been temporarily withdrawn from clinical use. Calcitonin plays a role in skeletal growth by suppressing bone resorption and stimulating bone formation ${ }^{2,47}$ and was found to be particularly beneficial in elderly patients with acute pain from vertebral fractures. The expert panels of both the FDA and European Medicines Agency recommended a suspension of clinical use due to the small risk of cancer compared to patients administered with a placebo. More research is necessary before calcitonin can be reintroduced as a therapy for osteoporosis.

\section{Romosozumalb}

Osteoporotic females treated with the human anti-sclerostin antibody romosozumab, which increases bone formation and reduces bone resorption, are provided with a reduced risk of vertebral fractures within one year. ${ }^{48}$ More research and particularly double blind clinical trials over extended periods are required to ascertain the clinical efficacy of the antibody and identify potential complications. Serious cardiovascular disease, recorded in 2.5\% of patients in phase three of the clinical trial (compared to 1.9\% in the control group treated with alendronate $)^{49}$ will influence the quest for FDA approval for clinical use in 2017.

\section{Human growth hormone}

Human growth hormone is released during childhood and exerts an anabolic effect on the skeleton through up-regulation of bone remodelling both directly and indirectly through mediation of insulin-like growth factor-1 (IGF-1). This results in increased osteoblastic and osteoclastic activities with net bone gain. 2,50 Great potential for the development of new therapeutic regimens targeting the diseased skeleton may be exposed through further research in this field.

\section{Calcilytics}

Calcilytics are a new class of bone-forming pharmaceuticals. They antagonise calcium receptors and evoke a short pulse of PTH release, thereby stimulating bone anabolism..$^{51}$ Their unfavourable pharmacokinetics, which could potentially be corrected, are presently delaying their entry to the market. ${ }^{52}$ 


\section{Conclusions}

The choice of medication for the prevention and management of skeletal deficiencies depends on factors such as affordability, tolerance, patient compliance and the medical history of the patient. Manufacturers' guidelines should be adhered to at all times. Short-term monitoring of treatment compliance and the skeletal response to medications that reduce resorption can be done through urinary $\mathrm{N}$-terminal telopeptide excretion which is significantly reduced with effective therapy. ${ }^{53}$ Long-term monitoring is performed through annual assessments of bone mineral density $(B M D)^{54}$ and if no response is recorded, alternative medication is recommended. The main factor which impacts on the clinical acceptance of most of the pharmaceuticals and autoantibodies manipulating skeletal health is the lack of placebo-controlled longterm clinical studies. Well-designed clinical trials over long periods of time are urgently needed to express the fracture reduction rates, clinical indications, complications and harm-to-benefit ratio of all prescribed drugs. ${ }^{55}$

\section{References}

1. Tabatabaei-Malazy $O$, Salari $P$, Khashayar P, Larijani B. New horizons in the treatment of osteoporosis. DARU J Pharm Sci 2017;25:2-16.

2. Raubenheimer E, Miniggio H, Lemmer L, van Heerden W. The role of bone remodelling in maintaining and restoring bone health: an overview. Clinic Rev Bone Miner Metab 2017:15:90-97.

3. Francis MB, Valent DJ. Historical perspectives on the clinical development of bisphosphonates in the treatment of bone diseases. J Musculoskelet Neuronal Interact 2007:7:2-8.

4. Bassett CAL, Donath A, Macagno F, Preisig R, et al. Diphosphonates in the treatment of myositis ossificans. Lancet 1969;2:845.

5. Smith R, Russell RGG, Bishop M. Diphosphonates and Paget's disease of bone. Lancet 1971:1:945-47.

6. Palma E, Correira JD, Campella MP, Santos I. Bisphosphonates as radionuclide carriers for imaging or systemic therapy. Mol Biosyst 2011;11:2950-66.

7. Colucci S, Minielli V, Zambonin G, Cirulli N, et al. Alendronate reduces adhesion of human osteoclast-like cells to bone and bone protein-coated surfaces. Calcif Tissue Int 1998;63:230-34.

8. Rodan GA, Fleisch HA. Bisphosphonates: mechanisms of action. J Clin Invest 1996:97:2692-96.

9. Plotkin LI, Lezcano V, Thostenson J, Weinstein RS, et al. Connexin 43 is required for the anti-apoptotic effect of bisphosphonates on osteocytes and osteoblasts in vivo. J Bone Miner Res 2008;23:1712-21.

10. Bellido T, Plotkin LI. Novel actions of bisphosphonates in bone: preservation of osteoblast and osteocyte viability. Bone 2011;49:50-55.

11. Zhou J, Ma X, Wang, T, Zhai S. Comparative efficacy of bisphosphonates in short term fracture prevention for primary osteoporosis: a systematic review with network meta-analyses. Osteoporos Int 2016;27:3289-300.

12. Rogers MJ, Gordon S, Benford HL. Cellular and molecular mechanisms of action of bisphosphonates. Cancer 2000;88:2961-78.

13. Crandall CJ, Newberry SJ, Gellad WG, et al. Treatment to prevent fractures in men and women with low bone density or osteoporosis:update of a 2007 report comparative effectiveness review No. 53. Agency for Healthcare Research and Quality. 2012; www.effectivehealthcare.ahrq.gov/repors/final/cfm

14. Kennel KA, Drake MT. Adverse effects of bisphosphonates: implications for osteoporosis management. Mayo Clin Proc 2009;84:632-38.

15. Singer FR, Bone HG, Hosking DJ, Lykes KW, et al. Paget's disease of bone: an endocrinological society clinical practice guideline. J Clin Endocrinol Metab 2014;99:4408

16. Hellstein J. Osteochemonecrosis: an overview. Head Neck Pathol 2014;8 482-90.

17. Schifter M, Coombs M, Georgio A, et al. Prevention of osteonecrosis of the jaw (ONJ) in patients on bisphosphonate therapies.2010; Department of Health, NSW, Sydney:Document GL2010_010, p1-24.

18. Cummings SP, San Martin J, McClung MR. Denosumab for prevention of fractures in postmenopausal women with osteoporosis. N Engl $\mathrm{J}$ Med 2009;361:756-62.

19. Lim SY, Bolster MB. Current approaches to osteoporosis treatment. Cur Opin Rhuematol 2015;27:216-24.

20. Rosen HN, Rosen CJ, Mulder JE. Denosumab for osteoporosis. Available at: www.uptodate.com. Accessed 7 June 2017

21. Pacifici R, Vannice JL, Rifas L et al. Monocytic secretion of interleukin-1 receptor antagonist in normal and osteoporotic women: effects of menopause and estrogen/progesterone therapy. J Clin Endocrinol Metab 1993;77:1135-41.

22. de Villiers TJ, Gass MLS, Haines CJ et al. Global consensus statement on menopausal hormone therapy. Climacteric 2013;16:203-204.

23. Felson DT, Nevitt MC. The effects of estrogen on osteoarthritis. Curr Opin Rheumatol 1998;10:269-72.

24. Rettner R. Hormone replacement therapy: types, benefits and risks. July 2014 www.livesciences.com.
25. de Villiers TJ, Pines A, Panay N, et al. International Menopause Society recommendations on menopause hormone therapy and preventative strategies for midlife health. Climacteric 2013;16:316-37.

26. Guidozzi F, Alperstein A, Bagratee JS et al. South African Menopause Society revised consensus position statement on menopausal hormone therapy, 2014. S A Med J 2014;104:537-43.

27. Gambacciani M, Levancini M. Hormone replacement therapy and the prevention of postmenopausal osteoporosis. PRZ Menopauzalny 2014;13:213-20.

28. Börjesson AE, Farman $\mathrm{HH}$, Moverare-Skrtic S, Engdahl C, et al. SERMs have substance specific effects on bone and these effects are mediated via ER $\alpha A F-1$ in female mice. Am J Phys Endocrinol Metab 2016;310:E912-8.

29. Mitlak BH, Cohen FJ. Selective estrogen receptor modulators. A look ahead. Drugs 1999;57:653-61.

30. Gennari L, Merlotti D, Nuti R. Selective estrogen receptor modulator (SERM) for the treatment of osteoporosis in post menopausal women: focus on lasofoxifene. Clin Interven Aging 2010;5:19-21.

31. Brőmme D, Panwar P, Turan S. Cathepsin Kosteoporosis trials, picnodysostosis and mouse deficiency models: commonalities and differences. Expert Opin Drug Discov 2016:22:1-16

32. Cheng ZW, Huang Z, Kuzuya M, et al.Cystein protease cathepsin in atherosclerosis-based vascular disease and its complications. Hypertension 2011;58:987-86

33. Mullard A. Merck \& Co. drops osteoporosis drug odanacatib. Nature Rev Drug Dis 2016;15:669. doi:10.1038/nrd.2016.207.

34. Neer RM, Arnauld JR, Zanchetta JR. Effect of parathyroid hormone (1-34) on fractures and bone mineral density in post-menopausal women with osteoporosis, New Engl J Med 2001;344:1434-41.

35. Weizman MN. The role of inflammatory cytokines, the RANKL/OPG axis, and the immunoskeletal interface in physiological bone turnover and osteoporosis. Scientifica 2013;2013:125705.

36. Tokuyama N, Hirose J, Omata $\mathrm{Y}$ et al. Individual and combining effects of anti-RANKL monoclonal antibody and teriparatide in ovarectomized mice. Bone Reports 2015;2:1-7.

37. Rizzoli R, Reginster J-Y, Boonen S, et al. Adverse reactions and drug interactions in the management of women with postmenopausal osteoporosis. Calcif Tissue Int 2011;89:91-104.

38. Bonnelye E, Chabadel A, Saltel F, et al. Dual effect of strontium ranelate: stimulation of osteoblast differentiation and inhibition of osteoclast formation and resorption in vitro. Bone 2008;42:129-38.

39. Atkins GJ, Weldon KJ, Halbout P, et al. Strontium ranelate treatment of human primary osteoblasts promotes an osteocyte-like phenotype while eliciting an osteoprotegerin response. Osteoporos Int 2009;20:653-64.

40. Brennan T, Rybchyn M, Green W, et al. Osteoblasts play key roles in the mechanisms of action of strontium ranelate. Br J Pharmacol 2009;157:1291300.

41. Hurtel-Lemaire AS, Mentaverri R, Caudrillier A. et al. The calcium sensing receptor is involved in strontium ranelate-induced osteoclast apoptosis. New insights into the associated signalling pathways. J Biol Chem 2009;284:575-84.

42. Marie PJ. Strontium ranelate: new insights into its dual mode of action. Bone 2007; $40:$ :S5-8.

43. Fonseca JE, Brandi ML. Mechanism of action of strontium ranelate:what are the facts? Clin Cases Min Bone Metab 2010;7:17-18

44. Meneur PJ, Roux C, Ortolani S, et al. Effects of long term strontium ranelate treatment on vertebral fracture risk in postmenopausal women with osteoporosis. Osteorporos Int 2009:10:1663073.

45. Kaufman JM, Audran M, Bianchi G, et al. Efficacy and safety of strontium ranelate in the treatment of osteoporosis in men. J Clin Endocrinol Metab 2013;98: 592-601.

46. Ducy P, Karsenty, G. Review. The two faces of serotonin in bone biology. J Cell Biol 2010;191:7-13

47. Cranney A, Welch $\mathrm{V}$, Adachi J, et al.Calcitonin for preventing and treating corticosteroid-induced osteoporosis. Cochrane Database Syst Rev 2001;1: Art. No.CD001983.

48. Cosman F. Crittenden DB, Jonathen D, et al. Romosozumab treatment in postmenopausal women with osteoporosis. New Engl J Med 2016:375:1532-43.

49. Amgen media release 5/21/2017. Accessed on 20 June 2017 at: www.amgen.com.

50. Olney RC. Regulation of bone mass by growth hormone. Med Paediatr Oncol 2003;41:228-34.

51. Rachner TD, Khosla S, Hofbauer LC. New horizons in osteoporosis. Lancet 2011;377:1276-87

52. Nemeth EF, Shoback D. Calcimimetic and calcilytic drugs for treating bone and mineral-related disorders. Clin Endocrin Metab 2013;27:373-84.

53. Palacios S, Neyro JL, Ferrer $\mathrm{J}$ et al. Reduction of urinary levels of $\mathrm{N}$-telopeptide correlates with treatment compliance in women with postmenopausal osteoporosis receiving alendronate. Menopause 2012;19:67-74.

54. Watts NB, Bilezikian JP, Camacho PM, et al. American Association of Clinica Endocrinologists Medical Guidelines for Clinical Practice for the diagnosis and treatment of postmenopausal osteoporosis. Endocrin Pract 2010;16:suppl 3: 1-37.

55. Erviti J, Gorricho J, Saiz LC, et al. Rethinking the appraisal and approval of drugs for fracture prevention. Front Pharmacol 2017;8:265. 